\title{
Solar Power Plant Detection on Multi-Spectral Satellite Imagery using Weakly-Supervised CNN with Feedback Features and m-PCNN Fusion
}

\author{
Nevrez Imamoglu*,+ \\ nevrez.imamoglu@aist.go.jp \\ Motoki Kimura* \\ kimura.motoki@aist.go.jp \\ Hiroki Miyamoto \\ miyamoto-hrk-tomeken@aist.go.jp \\ Aito Fujita \\ fujita.713@aist.go.jp \\ Ryosuke Nakamura \\ r.nakamura@aist.go.jp
}

\author{
Artificial Intelligence Research Center \\ National Institute of Advanced Industrial \\ Science and Technology \\ Tokyo, JAPAN
}

\begin{abstract}
Most of the traditional convolutional neural networks (CNNs) implement bottomup approach (feed-forward) for image classifications. However, many scientific studies demonstrate that visual perception in primates rely on both bottom-up and top-down connections. Therefore, in this work, we propose a CNN network with feedback structure for solar power plant detection on middle-resolution satellite images. To express the strength of the top-down connections, we introduce feedback CNN network (FB-Net) to a baseline $\mathrm{CNN}$ model used for solar power plant classification on multi-spectral satellite data. Moreover, we introduce a method to improve class activation mapping (CAM) to our FB-Net, which takes advantage of multi-channel pulse coupled neural network (mPCNN) for weakly-supervised localization of the solar power plants from the features of proposed FB-Net. For the proposed FB-Net CAM with m-PCNN, experimental results demonstrated promising results on both solar-power plant image classification and detection task.
\end{abstract}

\section{Introduction}

With the gradual increase in global warming of our planet Earth, investments on clean energy sources such as solar power plants have been increasing all over the world. For example, the photovoltaic capacity had expected to exceed 270 giga-watts worldwide between 2010 and 2016 as expressed in [1,2]. These solar power plants are visible through satellite imagery for allowing visual mapping and labelling of the plants. Since satellites have been collecting 


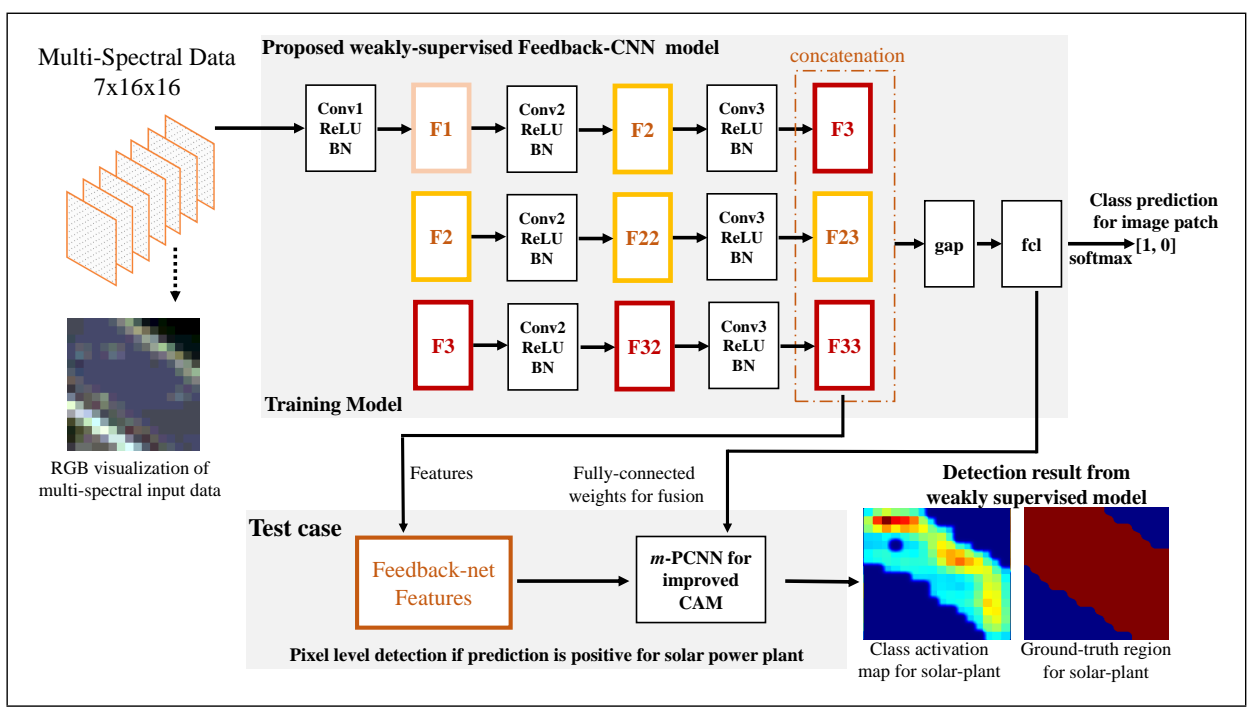

Figure 1: Training model: Unfolded representation of weakly supervised CNN with Feedback features (FB-Net) for solar power plant classification on multi-spectral imagery. The model includes three convolution layers (each convolution layer followed by ReLU and batch normalization) with feedback from features F2 and F3 to second layer, global average pooling layer (gap), and fully connected layer (fcl) Test case: Trained FB-Net features F3, F23, and F33 are used for class activation mapping (CAM). Instead of CAM in [17] as weighted linear combination, multi-channel Pulse Coupled Neural Network (m-PCNN) is used for feature fusion with fcl weights to obtain CAM.

data over years, it may also possible to observe temporal maps or labels regarding the change in clean energy development in solar power plants.

Recent advancements of deep learning [3-5] enabled researchers to develop many models, especially convolutional neural networks (CNNs), for classification or detection tasks on satellite imagery [2, 6-9]. However, despite the large number of works on object classification and detection in remote sensing, to the best of our knowledge, CNN model proposed by Ishii et al. [2] is the only base line work on solar power plant classification on a largescale (covering Japan) multi-spectral satellite data. Using Landsat- 8 data for Japan, the network [2] classifies small patches $(16 \times 16$ spatial resolution) and labels these patches with likelihood of being solar power plant. And, the system is running online on Land-browser interface [10]. However, detection by classification in [2] refers to labelling all pixels in the whole patch as the class prediction obtained from classification of the input data. So, we investigate pixel classification by using their model as a baseline to extend and improve solar power plant detection task. Certainly, current state-of-the-art models for recognition of remote sensing data such as Alex-Net [3] based CNN model OverFeat [9] can also be used by resizing $16 \times 16$ image patches to compatible input sizes for these models (e.g. $231 \times 231$ or $227 \times 227$ ). However, considering the model complexity of [3] and [9], this is not flexible and computationally more expensive for processing one Landsat- 8 scene with approximately $7500 \times 7500$ pixels. Also, as given in [2] and in our experimental results, model in [9] applied to solar power plant classification does not perform better than relatively simple model of Ishii et al. [2] CNN model (i.e. three convolution layers and a fully connected layer). 
On the other hand, conventional CNN implementations generally use feed-forward direct connections for layer structures to do classification and detection tasks [3-9]. However, as stated in [11], many scientific studies [12,13] on visual perception in primates have shown important signs of both bottom up and top-down connections in visual cortex areas. Inspired by these researches, CNN models with feedback unit or recurrent systems have been tried in some vision applications $[11,14-16]$ with the recent deep learning tools. These works $[11,14-16]$ use top-down information as modulator signal on the CNN layers or use them as a part of selective attention process to adjust neural activations between layers. However, despite being less plausible from the biological perspective, another way of implementing feedback on CNN is to use higher contextual features as input to lower CNN layers, and then, apply feed-forward direct computation. With these feedback structure, it is possible to obtain richer feature representation (e.g. more class dependent feature activations) before the decision layer by providing relatively low-level and high level contextual features. Therefore, in this work, we describe a CNN model based on feedback features to achieve solar power plant classification and detection on multi-spectral imagery.

In addition, as the data-set shared by [2] does not include pixel-wise annotation for training data, it is not possible to try fully-supervised models such as FCN [5] or similar end-toend pixel-wise training models. Therefore, we investigate the use of localization methods on weakly-supervised CNNs through taking advantage of class activations map approaches such as CAM [17]. However, we consider that class dependent activation of a pixel should consider the activations around the surrounding pixels while fusing feature channels with respect to weights learned or obtained from the CNN model. Therefore, to improve the detection performance of class activation maps, we propose the use of pulse-coupled neural networks (PCNN) for feature fusion by integrating it as an improved CAM approach. PCNN feature fusion not only considers weighted average of channels but also it considers activations of each pixel while taking surround pixel activations into consideration.

\section{With the stated purpose, our contributions in this paper are the following:}

i) We propose a feedback network (Fig.1) for solar power plant classification on middle-resolution (30 meters/pixel) satellite images. To express the strength of the top-down connections, we introduce feedback structure (FB-Net) to Ishii et al. [2] baseline CNN model (I-Net) used for solar power plant classification.

ii) As given in Fig.1, we use global average pooling for features before fully connected layer as in [17] to obtain weights in feature fusion for weakly-supervised class activation mapping $[17,18]$. However, in test phase, we propose use of multi-channel PCNN (m-PCNN) [19-21] based feature fusion to improve class activation mapping (CAM) $[17,18]$ to our FB-Net as a weakly-supervised localization of class dependent features for solar power plants. m-PCNN provides improved feature fusion due to spatial connections of the pixels, which effects final pixel class activations.

For image patch classification performance evaluation, we use Intersection over Union (IoU) metric as in [2]. For detection performance of the proposed m-PCNN based CAM model with our FB-Net, we make quantitative validation by doing analysis and comparison on variations of I-Net [2] and our proposed FB-Net, which are demonstrated in experimental results. To do so, we apply simple feature averaging, CAM [17], and Gradient based CAM [18] on CNN features for both I-Net [2] and our FB-Net. As detection evaluation metric for class activation maps, we use Area Under Curve (AUC) computed from Receiver Operating 
Characteristic (ROC) curve. ROC curve is obtained through applying various thresholds to the activation maps. For the proposed FB-Net CAM with m-PCNN (Fig.1), experimental results demonstrated promising results on both solar power plant classification and detection task based on the IoU and AUC metrics respectively.

The outline of the paper follows: Section 2 is the brief explanation for the data-set used in this work, Section 3 describes the proposed weakly-supervised FB-Net-CAM with m-PCNN integration for classification and detection, Section 4 demonstrates the experimental results, finally, some concluding remarks are given.

\section{Dataset}

To train and test the models, we use the data-set which was introduced and provided by Ishii et al. [2]. For this data-set, publicly available multi-spectral satellite images captured by Landsat 8 and power plant database are used. In this section, we explain the overview of data-set from Landsat 8 imagery. Landsat 8 is an American Earth observation satellite which has been operated since 2013 [2, 22, 23]. It observes whole surface of the Earth with 11 bands of different wavelength and spatial resolutions [22, 2]. As explained in [2], we also used the first 7 bands having same spatial resolution and mostly no overlap in wavelength. The entire data-set was constructed from 20 satellite images of Japan captured in 2015, and each of the images captures roughly an area of $170 \mathrm{~km} \times 183 \mathrm{~km}$ [2]. Because the Landsat- 8 $[22,23]$ satellite image is too coarse for small solar panels (e.g. solar panels on the roof of a house ) or relatively small scale solar power plants, such solar panels are not visible clearly or feasible for manual labelling for generating training and test data. Therefore, the authors in [2] create positive samples if only the power plants whose output is greater than $5 \mathrm{MW}$ [2]. Then, solar power plants are manually annotated with a polygon in the satellite images. Then, small patches with $16 \times 16$ spatial resolution are extracted. A sample patch is labelled as mega-solar if it covers more than $20 \%$ solar panel area obtained by the manually drawn polygons [2]. Then, to use these satellite imagery, each imagery was divided into cells with the size of $16 \times 16$ pixels with their respective ground truth class labels (solar power plant or not). With these conditions, we obtained the training and test data as image patches and class labels from [2] with sample numbers: i) Training data: Positive Sample are 4,851 and Negative Samples are 320,000, i) Test data: Positive Sample are 105 and Negative Samples are 820,666 .

\section{Proposed Method}

\subsection{Feedback CNN Model for Solar Power Plant Classification}

In this paper, we propose a model which has feedback paths by using I-Net (Ishii et al. [2]) as the baseline network. We refer to our model as FB-Net in this paper. FB-Net has 3 convolutional layers followed by a fully connected layer like I-Net but it has 2 feedback paths inside the model as shown in Fig.1. The features output from the second convolutional layer (F2 features in Fig.1) goes back to the second convolutional layer and then goes through the third convolutional layer. This feedback path is illustrated as the middle path of the trained model in Fig.1. Likewise, the features output from the third convolutional layer (F3 features in Fig.1) goes through the second convolutional layer and then the third convolutional layer again. This feedback path is the bottom path of the trained model illustrated in Fig.1. The 
features output from the top, middle, and bottom paths (see Fig.1, F3, F23, and F33 features respectively) are all concatenated before the fully connected layer. Note that all the second convolutional layers shown in Fig. 1 share the same weights as well as the third convolutional layers. These features are processed by global average pooling followed by a fully connected layer that output the prediction result for classification task with soft-max.

\subsection{Solar Power Plant Detection with Weakly Supervised Feedback-Net and m-PCNN based Class Activation Mapping}

I-Net in [2] uses pixel-level labelling of solar power plants results from classification. So, if an input data with $16 \times 16$ spatial resolution is predicted as solar power plant, detection by classification in [2] assigns labels to all pixels covering $480 \mathrm{~m} \times 480 \mathrm{~m}$ as part of solar power plants. However, this very coarse labelling cannot result in reliable accuracy because one pixel is around 30 meters on Landsat- 8 multi-spectral data even though it may show reasonable and acceptable solar-power plant areas on a data with very large spatial resolution. In addition, approaches such as CAM and Grad-CAM for class dependent feature fusion does not consider the activations of surrounding regions which may result in false positives. Fusion methods for the feature maps or class activation maps should consider not only the pixel to pixel integration of the same locations but also the relation around the surrounding pixels regarding the class dependent activations on each feature channel $[19,20]$. However, fusion of class dependent activations are generally based on mean value, weighted averaging, maximum of the same location over feature channels so connections or relations of surrounding pixels with high activations can be missing during fusion in these approaches. Therefore, we introduce a weakly supervised solar power plant detection framework by taking advantage of pulse-coupled neural network (PCNN) based class activation mapping.

$\mathrm{PCNN}$ is a promising algorithm on information fusion. As an unsupervised neural network model, PCNN demonstrated its usability in various image processing applications such as information fusion, segmentation, etc. [19-21]. PCNN algorithm uses Eckhorn model introducing model for the cat visual cortex [24]. This model is modified for PCNN to use in image processing applications [24, 19-21]. In this paper, we take advantage of multi-channel PCNN (m-PCNN) model proposed by [19], and we apply m-PCNN for feature fusion to create class activation map for solar power plant detection. Iterative process of $\mathrm{m}-\mathrm{PCNN}$ (see Fig.2) can be formulated through Eq.1 to Eq.4 [19, 20, 24]. As stated in [20, 19], m-PCNN based feature fusion can help to create class activation maps with improved local uniformity and continuity around pixels with high class dependent activations (e.g. solar power plant regions).

$$
\begin{gathered}
H_{i, j}^{k}[n]=e^{-\alpha_{H}} H_{i, j}^{k}[n-1]+V_{H}\left(\mathbf{W} * Y_{i, j}[n-1]\right)+S_{i, j}^{k} \\
W_{i, j}=\frac{1}{\left(\left(i-c_{x}\right)^{2}+\left(j-c_{x}\right)^{2}\right)^{\frac{1}{2}}}, \operatorname{and} W_{c_{x}, c_{y}}=0 \\
U_{i, j}[n]=\prod_{k=1}^{K}\left(1+\beta^{k} H_{i, j}^{k}[n]\right) \\
Y_{i, j}[n]= \begin{cases}1, & U_{i, j}[n]>T_{i, j}[n-1] \\
0, & \text { otherwise }\end{cases} \\
T_{i, j}[n]=e^{-\alpha_{T}} T_{i, j}[n-1]+V_{T} Y_{i, j}[n]
\end{gathered}
$$


where $k=\{1, \ldots, 96\}$ refers to the input channels (CNN features extracted from FB-Net; i.e. F3, F23, and F33 in Fig.1 are concatenated), $\mathbf{H}$ is the external stimulus from the feed function in (Eq.1) with input stimulus $\mathbf{S}, \beta^{k}$ is the weight showing the importance of the $k^{\text {th }}$ data channel, $\mathbf{W}$ is the constant synaptic linking weights, "*" is the convolution operation, $\mathbf{U}$ is the combination of feeding and linking process, $\mathbf{U}$ also expresses the internal state of the neuron at iteration $\mathrm{n}, \mathbf{Y}$ is the fired neurons that are defined by the dynamic threshold $\mathbf{T}$, $V_{H}$ and $V_{T}$ are used as scaling parameters, and $\alpha^{H}$ and $\alpha^{H}$ are the time constants. When the all neurons (pixels) are processed through the fusion of Feedback-Net CNN features, the iteration process from Eq. 1 to 4 terminates. And, $\mathbf{U}$ at the last iteration is the fusion result as our output for solar power plant detection. $\mathbf{H}$ and $\mathbf{S}$ are initialized as the feature channels.

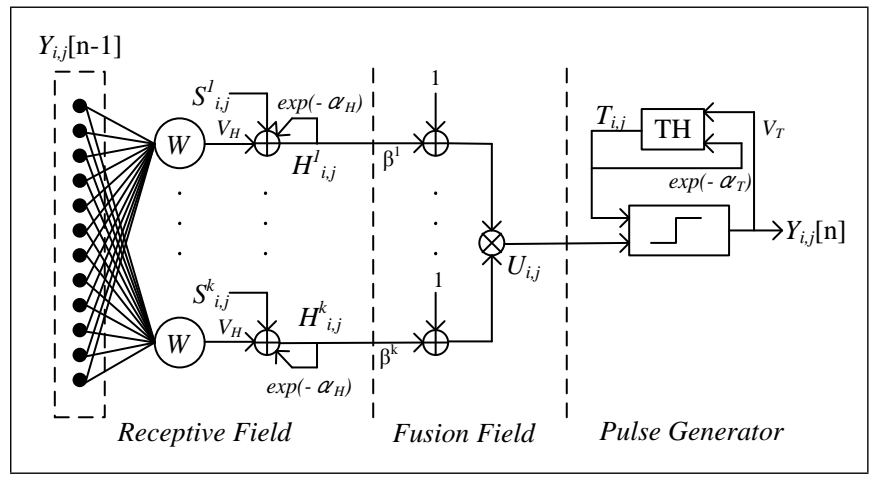

Figure 2: Structure and work flow of an m-PCNN neuron

To use m-PCNN to obtain class activation map on CNN features, we adaptively assign weighting factor $\beta_{k}$ based on the class dependent weights obtained from the fully connected layer (see Fig.1) connected to global average pooling [17]. Proposed FB-Net CAM with mPCNN results in very promising detection results. Section 4 shows the performance analysis of the proposed classification and detection tasks on test data.

\section{Experiments}

Batch normalization and drop-out layers: We train the models by using the data-set of $16 \times 16$-pixel image patches with corresponding annotations using cross-entropy loss. We use Batch Normalization layers [25, 2] after ReLU function of each convolution layer as in [2] (see Fig.1).

Batch Normalization layers are only used and updated during training, and these layers become fixed linear transformations in test phase. As in [2], we also use a Dropout layer [26] that sets the output after the third convolution layer and before the fully connected layer to 0 with $50 \%$ possibility, independently at each pixel of the output. This layer reduces overfitting of the models to the training data $[2,26]$. We use SGD (Stochastic Gradient Descent) with fixed learning rate of 0.01 and momentum of 0.9 to optimize weights of the models.

Balanced mini-batch sampling: As described in [2], despite augmenting the positive samples and sub-sampling the negative samples, training data-set still has a large bias between positives and negatives. While training the model, we get mini-batches with the size of 128 
samples from the training set [2]. Each mini-batch consists of 8 positives and 120 negatives so that there are always positive and negative samples for every mini-batch [2]; therefore, with this balanced sampling of negative and positive samples, we prevent the model training from being biased by negative samples despite the large number.

\subsection{Experimental Results for Classification}

In this section, we compare solar-power plant classification performance (IoU metric) of proposed Feedback-Net (see Fig.1) with I-Net [2]. We also added CNN model of Penatti et al. [9] and Support Vector Machine (SVM) based classification results demonstrated in [2]. In addition, we also tried I-Net with global average pooling (gap) for CAM and our FB-Net without gap layer to see if classification accuracy is decreasing on I-Net and FB-Net by adding GAP as referred in CAM paper [17]. After training each CNN model up to 4,000 epochs, we evaluate each model in both classification and detection accuracy with the best performing epoch based on intersection over union (IoU) for each model respectively using test data with 105 positive and 802666 negative samples of image patches.

Regarding solar power plant classification performance, comparison of proposed FB-Net to SVM in [2], Penatti [9], I-Net [2], I-Net with gap, and our FB-Net without gap are given in Table 1. For comparison, we used Intersection over Union (IoU), where IoU $=$ True Positive / (True Positive + False Negative + False Positive). It can be used in comparisons of models instead of accuracy, especially when background samples (negative samples or pixels) are much bigger than the foreground samples (positive samples or pixels) in test data. Certainly, other metrics such as Precision - Recall - F-measure can also be used for evaluation but in reference [2] only IoU results were presented for I-Net [2], SVM, and Penatti [9]. And we do not have results for Precision and Recall on SVM and Penatti [9] models given in [2] so we also applied IoU to compare models.

It should be noted that our test results on I-Net [2], I-Net+gap, FB-Net w/o gap, and proposed FB-Net (Fig.1) are results directly using training model without validation data, which can help to find optimal threshold on soft-max output value for decision. Main usage of validation is generally to decide when to stop network training by avoiding over-fitting. But, in [2], decision threshold of network scores are adjusted with validation giving IoU 0.52. It is also possible to use finding optimal decision threshold on class score values but the way of finding threshold (limited number of thresholds were applied, and then best one was selected based on IoU) is not very good and number of validation positive samples in [2] are very small (only 21 samples).

Even though I-Net [2] yields 0.52 IoU performance in [2] when validation data is used, which is outperforming SVM and Penatti [9] model for solar-power plant classification, it is hard to state or find optimal threshold on class scores for classification with given number of samples and method. Therefore, we did not see it a very reliable way, and decided not

\begin{tabular}{|l|c|c|c|c|c|c|}
\hline & SVM [2] & Penatti [9] & I-Net [2] & I-Net+gap [2] & FB-Net w/o gap & Proposed FB-Net \\
\hline \hline IoU & 0.23 & 0.39 & 0.36 & 0.30 & 0.34 & $\mathbf{0 . 5 6}$ \\
TP rate & n/a & n/a & 0.923810 & 0.933333 & 0.923810 & 0.838095 \\
TN rate & n/a & n/a & 0.999796 & 0.999722 & 0.999779 & 0.999935 \\
\hline
\end{tabular}

Table 1: Intersection over Union based performance comparison of proposed FB-Net with SVM [2], Penatti [9], I-Net [2], I-net with gap layer, and our FB-Net without gap layer 
to use validation on training and experiments. We can improve classification accuracy even more by using validation data to decide decision threshold on the model predictions for improved performance; however, we wanted to show that proposed model can outperform the compared models SVM, Penatti [9], and I-Net improved with validation data. In addition, in this work, we would like to focus more on the detection performances of these models for the true positive cases, which is explained in the following section.

In our experiments, I-Net [2] accuracy decreased when gap layer is added to the network for obtaining CAM, which is also an observation of [17] proposing CAM approach. On the other hand, interestingly, our proposed FB-Net has better IoU performance than FB-Net without gap model. Since the training and test data is largely biased to negative samples, very small difference, or error on True Negative rate, may result in a significant change on the IoU result despite having high classification accuracy both on True Positive (TP) and True Negative (TN) rate. Therefore, IoU performance highly dependent on the accuracy of TN rate compared to TP rate. For example, proposed FB-Net demonstrated the best performance regarding IoU because it has the highest TN rate regardless of its lower TP rate accuracy compared to other approaches. However, FB-Net without gap layer has worse IoU performance than both original I-Net [2] without gap layer and proposed FB-Net using gap layer.

\subsection{Experimental Results for Detection}

We compared the detection performance of proposed Feedback-Network (FB-Net integrated with m-PCNN based feature fusion in Fig.1) to CAM [17] and Grad-CAM [18] models applied to I-Net [2] and our FB-Net. For an unbiased comparison among the models, we calculated the detection accuracies of models listed below if and only if all the models' classification predictions are true positive for the given test data (i.e. multi-spectral test data with $7 \times 16 \times 16$ is predicted as mega-solar power plant). Compared models for solar power plant class activation mapping : 1) I-Net feature-averaging [2], 2) I-Net using CAM in [17], 3) I-Net using Grad-CAM [18], 4) feature averaging on FB-Net w/o gap layer, 5) FBNet using CAM in [17], 6) FB-Net w/o gap layer using Grad-CAM in [18], 7) Proposed FB-Net with m-PCNN based CAM (see Fig.1 test case), 8) FB-Net w/o gap layer with m-PCNN based Grad-CAM.

Among the 105 positive samples in test data (including solar power plant regions), 86 of them are predicted as solar power plant class for all the eight models compared in above list; therefore, for detection evaluation, we used these 86 samples. Class activation map results used to evaluate detection performances can be seen in Fig.3. For better visualization and seeing effect of m-PCNN fusion, feature representations are up-sampled with a scale factor of 16 using nearest neighbour assignment so that the detection results in Fig.3 are processed in 256x256 for all approaches including m-PCNN with linking size 15 for W (Eq.1).

For evaluation, we use Area Under Curve (AUC) metric [27, 28]. AUC is the area under the Receiver Operating Characteristic (ROC) curve, and ROC is curve of true positive rate (TPR) with respect to false positive rate (FPR) of detection (e.g. class activation maps) by changing the threshold value to compare with ground truth maps [27, 28]. AUC and ROC results of the compared models are given in Fig.4. Proposed model in Fig.1, m-PCNN integration for class activation mapping to FB-Net, outperforms all other detection approaches applied to I-Net or FB-Net. The lowest detection performance is obtained from I-Net Cam model (AUC value is 0.6652). And, our proposed FB-Net with m-PCNN using the CAM weights (see Fig.1) has the best AUC performance (0.9571) for the given test data. In gen- 


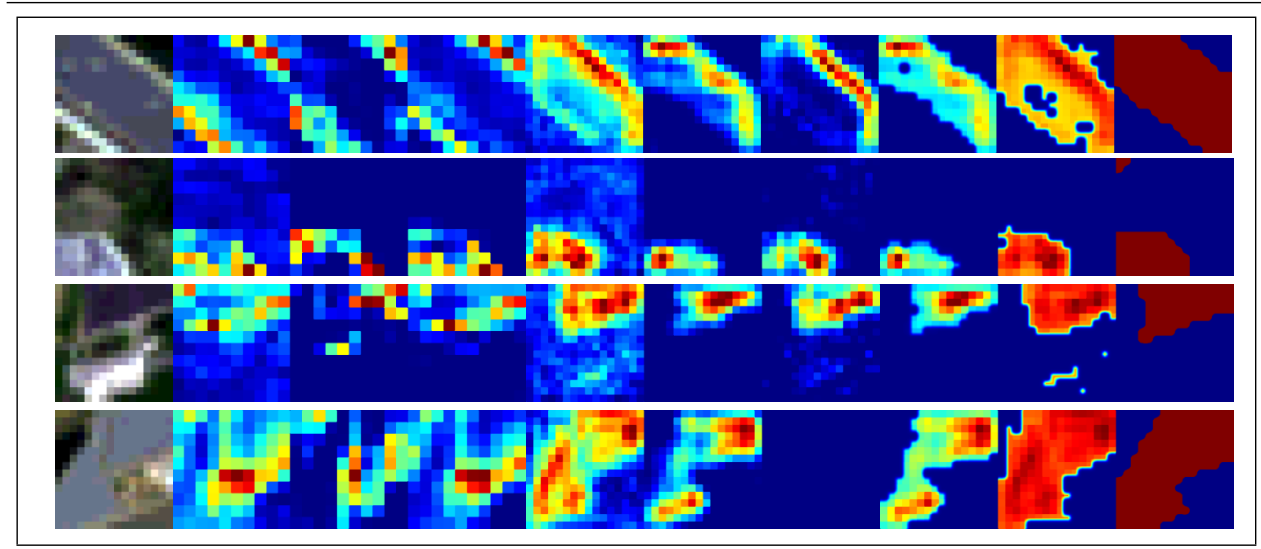

Figure 3: Solar power plant detection results of (a) sample images (RGB visualization of multi-spectral data) (b) I-Net [2] feature averaging, (b) I-Net+gap, (c) I-Net using GradCAM in [18], (d) feature averaging on FB-Net w/o gap, (e) FB-Net using CAM in [17], (f) FB-Net w/o gap using Grad-CAM [18], (g) Proposed FB-Net with m-PCNN based CAM, (h) FB-Net w/o gap layer with m-PCNN based Grad-CAM, (i) ground-truth

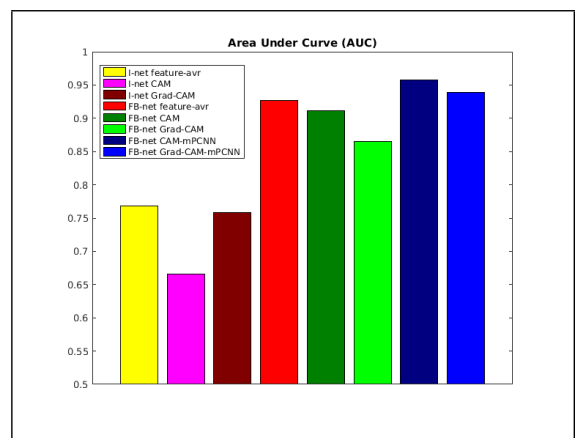

(a)

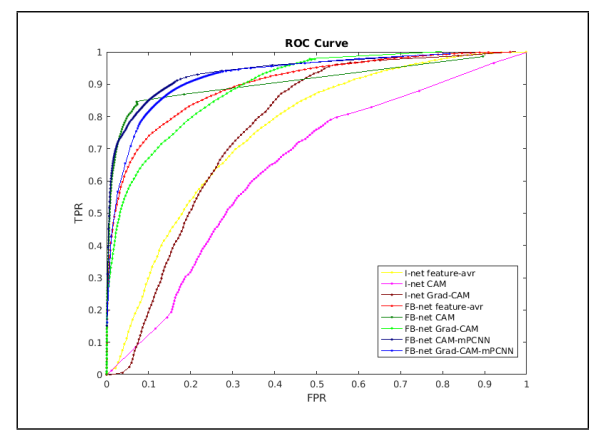

(b)

Figure 4: (a) Area Under Curve results for the compared models obtained from their respective (b) ROC curves

eral, FB-Net based detection results outperform I-Net base approaches. Grad-CAM performances decreases because in a very few test samples, Grad-CAM computation for both I-Net and FB-Net yields zero gradient during backward process due to very small precision. In our system, GPU computation using Chainer Deep Learning library supports float32 bit precision. So, detection fails in these cases (see last column in Fig.3) when the gradient cannot be represented with float 32 bit precision.

An interesting observation is that simple feature averaging performs better than common CAM [17] and Grad-CAM [18] on both I-Net and FB-Net. In a binary classification, activations of $\mathrm{CNN}$ features at the final convolutional layer seems to be very representative for the object of interest (e.g. solar power plants). Therefore, learned features result in quite reliable detection performance on test data, especially for our proposed FB-Net. These detection based performance evaluation of FB-Net demonstrate that using higher level features as a top-down feedback signal with the same bottom-up process in the same network (using 
shared weights as in Fig.1) can help richer representation of the class dependent activations. Therefore, it can yield better class activations around object region from the CNN features of the last convolutional layers.

\section{Conclusion}

In this work, we introduced a weakly supervised Feedback CNN to classify and detect solar power plants on multi-spectral data. For this, we took advantage of class activation mapping with m-PCNN on the proposed Feedback-CNN model, which can also be applied to larger scale multi-spectral scene (see sample results in Fig.5) without any resizing process. Experimental results demonstrated that $\mathrm{CNN}$ features extracted from the FB-Net are satisfactory for pixel-wise detection task (AUC metric) while providing good accuracy on classification based on IoU metric.

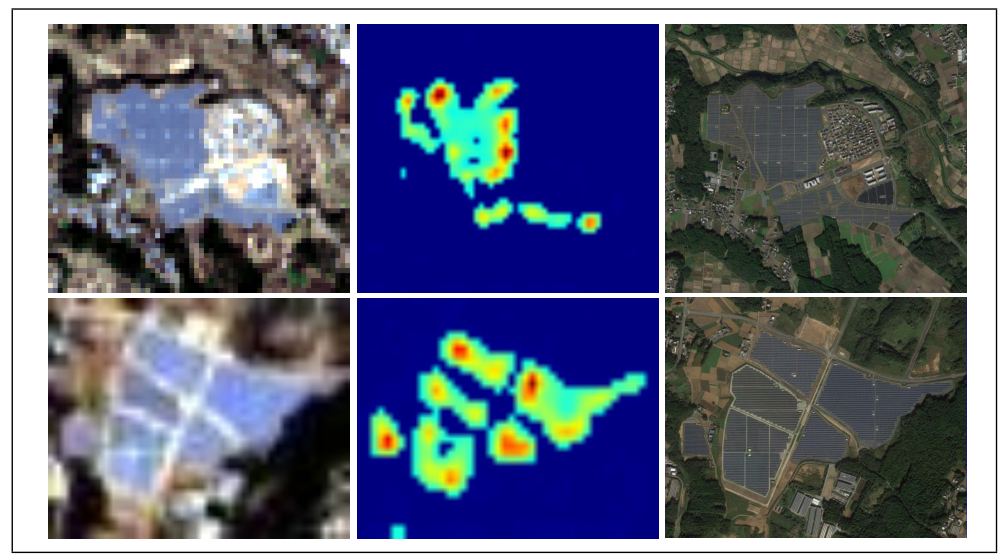

Figure 5: (left) RGB visualization of input data (middle) CAM results obtained from proposed model in Fig.1 (right) Google Earth view of same area for reference

Generalization of feedback feature fusion to other models and tasks are important. Therefore, as a future work, we are planning to try other baseline models to FB-Net and also try world-wide tests rather than limited region (Japan). Furthermore, we would like to extend the FB-Net implementation to other satellite imagery tasks such as change detection, damage detection, etc.

In addition, we can investigate the effect of linking size in $\mathrm{m}-\mathrm{PCNN}$ on detection accuracy. Instead of setting parameters empirically, these parameters can also be optimized with greedy search, genetic algorithm, or Bayesian optimization models.

\section{Acknowledgements}

This paper is based on results obtained from a project commissioned by the New Energy and Industrial Technology Development Organization (NEDO). 


\section{References}

[1] IHS, Inc., "As European Solar Installations Slow, China, US and Japan Lead Global Installed PV Capacity in 2016, IHS Says," http://press.ihs.com/press-release/europeansolar-installations-slow-china-us-and-japan-lead-global-installed-pv-capacity, 2016, [Online; accessed March 2017].

[2] T. Ishii, , E. Simo-Serra, S. Iizuka, Y. Mochizuki, A. Sugimoto, H. Ishikawa, and R. Nakamura. Detection by Classification of Buildings in Multispectral Satellite Imagery. Int. Conf. on Pattern Recognition (ICPR), 2016.

[3] J. A. Krizhevsky, I. Sutskever, and G. E. Hinton. Imagenet classification with deep convolutional neural networks. Conf. on Neural Information Processing Systems (NIPS), 2012.

[4] K. Simonyan and A. Zisserman. Very Deep Convolutional Networks For Large Scale Image Recognition. Int. Conf.On Learning Representations (ICLR), 2014.

[5] J. Long, E. Shelhamer, and T. Darrell. Fully convolutional networks for semantic segmentation. Int. Conf on Computer Vision and Pattern Recognition (CVPR), 2015.

[6] F. Hu , G.-S. Xia, J. Hu and L. Zhang. Transferring Deep Convolutional Neural Networks for the Scene Classification of High-Resolution Remote Sensing Imagery. Remote Sensing, Vol.7, No.11, 2015.

[7] J. M. Castelluccio, G. Poggi, C. Sansone, and L. Verdoliva, "Land use classification in remote sensing images by convolutional neural networks," arXiv preprint arXiv:1508.00092 , 2015.

[8] I. Sevo and A. Avramovic. Convolutional Neural Network based Automatic Object Detection on Aerial Images. IEEE Geoscience and Remote Sensing Letters, Vol.3, No.5, 2016.

[9] O. A. B. Penatti, K. Nogueira, and J. A. dos Santos, "Do deep features generalize from everyday objects to remote sensing and aerial scenes domains?" Int. Conf on Computer Vision and Pattern Recognition (CVPR) Workshops, 2015.

[10] LandBrowser online available at: legacy.geogrid.org/solar/

[11] M. Stollenga, J. Masci, F. Gomez, J. Schmidhuber. Deep Networks with Internal Selective Attention through Feedback Connections. Conf. on Neural Information Processing Systems (NIPS), 2014.

[12] D. J. Felleman and D. C. van Essen. Distributed hierarchical processing in the primate cerebral cortex. In: Cerebral Cortex vol.1, no.1, pp.1-47, 1991.

[13] J. Bullier. Hierarchies of Cortical Areas. In: The Primate Visual System . Ed. by J.H. Kaas and C.E. Collins. New York: CRC Press, pp.181-204, 2004.

[14] C. Sao et.al. Look and Think Twice: Capturing Top-Down Visual Attention with Feedback Convolutional Neural Networks. Int. Conf. on Computer Vision (ICCV) 2015. 
[15] X. Liu, A. Zhang, T. Tiecke, A. Gros, T. S. Huang. Feedback Neural Network for Weakly Supervised Geo-Semantic Segmentation. arXiv preprint arXiv:1612.02766, 2016.

[16] K. Xu et.al. Show, Attend and Tell: Neural Image Caption Generation with Visual Attention. arXiv preprint arXiv: 1502.03044, 2015.

[17] B. Zhou, A. Khosla, A. Lapedriza, A. Oliva, A. Torralba. Learning Deep Features for Discriminative Localization. Int. Conf on Computer Vision and Pattern Recognition (CVPR), 2016.

[18] R. R. Selvaraju, M. Cogswell, A. Das, R. Vedantam, D. Parikh, D. Batra. Grad-CAM: Visual Explanations from Deep Networks via Gradient-based Localization. arXiv preprint arXiv:1610.02391, 2016.

[19] Z. Wang and Y. Ma. Medical image fusion using m-PCNN. Information Fusion, vol. 9, pp.176-185, 2008.

[20] Nevrez Imamoglu, Zhixuan Wei, Huangjun Shi, et al., Saliency Fusion in Eigenvector Space with Multi-Channel Pulse Coupled Neural Network, arXiv preprint arXiv:1703.00160, 2017.

[21] S. Liu, D. He and X. Liang. An improved hybrid model for automatic salient region detection. IEEE Signal Processing Letters, vol.19, no.4, pp.207-210, 2012.

[22] D. Roy et.al. Landsat-8: Science and product vision for terrestrial global change research. Remote Sensing of Environment, vol. 145, pp.154-172, 2014.

[23] Landsat-8: https://landsat.usgs.gov/landsat-8 , [Online accessed March 2017].

[24] T. Lindblad and M. Kinser, Image processing using pulse coupled neural networks. 2nd Revised ed., Springer, 2005, ch.1-2.

[25] S. Ioffe, C. Szegedy. Batch Normalization: Accelerating Deep Network Training by Reducing Internal Covariate Shift. arXiv preprint arXiv: 1502.03167, 2015.

[26] N. Srivastava, G. Hinton, A. Krizhevsky, I. Sutskever, R. Salakhutdinov; Dropout: A Simple Way to Prevent Neural Networks from Overfitting. Journal of Machine Learning Research, vol.15, pp.1929-1958, 2014.

[27] T. Liu, J. Sun, N.-N. Zheng, X. Tang, H.-Y. Shum. Learning to detect a salient object. IEEE Conf. on Computer Vision and Pattern Recognition (CVPR ), 2007.

[28] Y. Fang, Z. Chen, W. Lin, C.-W. Lin. Saliency detection in the compressed domain for adaptive image retargeting. IEEE Transactions on Image Processing, vol. 21, no. 9, pp. 3888-3901, 2012. 